\title{
Synchronous Spike Patterns in Macaque Motor Cortex during an Instructed-Delay Reach-to-Grasp Task
}

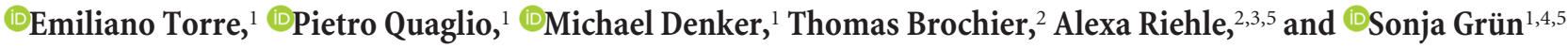 \\ ${ }^{1}$ Institute of Neuroscience and Medicine and Institute for Advanced Simulation and JARA Brain Institute I, Jülich Research Centre, 52425 Jülich, Germany, \\ ${ }^{2}$ Institut de Neurosciences de la Timone, UMR 7289, CNRS, Aix-Marseille Université, 13005 Marseille, France, ${ }^{3}$ Institute of Neuroscience and Medicine, \\ Jülich Research Centre, 52425 Jülich, Germany, ${ }^{4}$ Theoretical Systems Neurobiology, RWTH Aachen University, 52062 Aachen, Germany, and ${ }^{5 R I K E N ~ B r a i n ~}$ \\ Science Institute, 351-0198 Wako Shi, Japan
}

The computational role of spike time synchronization at millisecond precision among neurons in the cerebral cortex is hotly debated. Studies performed on data of limited size provided experimental evidence that low-order correlations occur in relation to behavior. Advances in electrophysiological technology to record from hundreds of neurons simultaneously provide the opportunity to observe coordinated spiking activity of larger populations of cells. We recently published a method that combines data mining and statistical evaluation to search for significant patterns of synchronous spikes in massively parallel spike trains (Torre et al., 2013). The method solves the computational and multiple testing problems raised by the high dimensionality of the data. In the current study, we used our method on simultaneous recordings from two macaque monkeys engaged in an instructed-delay reach-to-grasp task to determine the emergence of spike synchronization in relation to behavior. We found a multitude of synchronous spike patterns aligned in both monkeys along a preferential mediolateral orientation in brain space. The occurrence of the patterns is highly specific to behavior, indicating that different behaviors are associated with the synchronization of different groups of neurons ("cell assemblies"). However, pooled patterns that overlap in neuronal composition exhibit no specificity, suggesting that exclusive cell assemblies become active during different behaviors, but can recruit partly identical neurons. These findings are consistent across multiple recording sessions analyzed across the two monkeys.

Key words: cell assemblies; massively parallel spike trains; motor cortex; spike synchrony; temporal coding

Significance Statement

Neurons in the brain communicate via electrical impulses called spikes. How spikes are coordinated to process information is still largely unknown. Synchronous spikes are effective in triggering a spike emission in receiving neurons and have been shown to occur in relation to behavior in a number of studies on simultaneous recordings of few neurons. We recently published a method to extend this type of investigation to larger data. Here, we apply it to simultaneous recordings of hundreds of neurons from the motor cortex of macaque monkeys performing a motor task. Our analysis reveals groups of neurons selectively synchronizing their activity in relation to behavior, which sheds new light on the role of synchrony in information processing in the cerebral cortex.

\section{Introduction}

The computational relevance of spike synchronization at millisecond precision among neurons in the cerebral cortex has been hotly debated during the last two decades. The efficacy of syn-

\footnotetext{
Received Dec. 2, 2015; revised May 13, 2016; accepted June 18, 2016.

Author contributions: E.T., P.Q., M.D., T.B., A.R., and S.G. designed research; E.T., P.Q., T.B., and A.R. performed research; E.T., P.Q., and M.D. contributed unpublished reagents/analytic tools; E.T. and P.Q. analyzed data; E.T., P.Q., M.D., T.B., A.R., and S.G. wrote the paper.

This work was supported by Helmholtz Portfolio Supercomputing and Modeling for the Human Brain, BrainScaleS (EU Grant 269912), Human Brain Project (EU Grant 604102), and Collaborative Research Agreement RIKENCNRS, ANR GRASP. We thank Ivan Balansard for surgical help, the INT animal house team for animal care, Joel Baurberg and Xavier Degiovanni for technical assistance, and Lyuba Zehl for help in managing the meta-data and for providing Figure 1 in this manuscript.

This article is freely available online through the J Neurosci Author Open Choice option.
}

chronous compared with asynchronous input in triggering a spike response was explored in theoretical studies that led to the notion of the coincidence detector (Abeles, 1982; Rudolph and Destexhe, 2003) and was supported by experimental evidence (Softky and Koch, 1993; Azouz and Gray, 2000, 2003). Pairwise or low-order correlations have been shown to be related to be-

Correspondence should be addressed to Sonja Grün, Institute of Neuroscience and Medicine and Institute fo Advanced Simulation and JARA Brain Institute I, Jülich Research Centre, Wilhelm-Johnen-Straße, 52425 Jülich, Germany. E-mail: s.gruen@fz-juelich.de.

D0I:10.1523/JNEUROSCI.4375-15.2016

Copyright $\odot 2016$ Torre, et al.

This is an Open Access article distributed under the terms of the Creative Commons Attribution License Creative Commons Attribution 4.0 International, which permits unrestricted use, distribution and reproduction in any medium provided that the original work is properly attributed. 


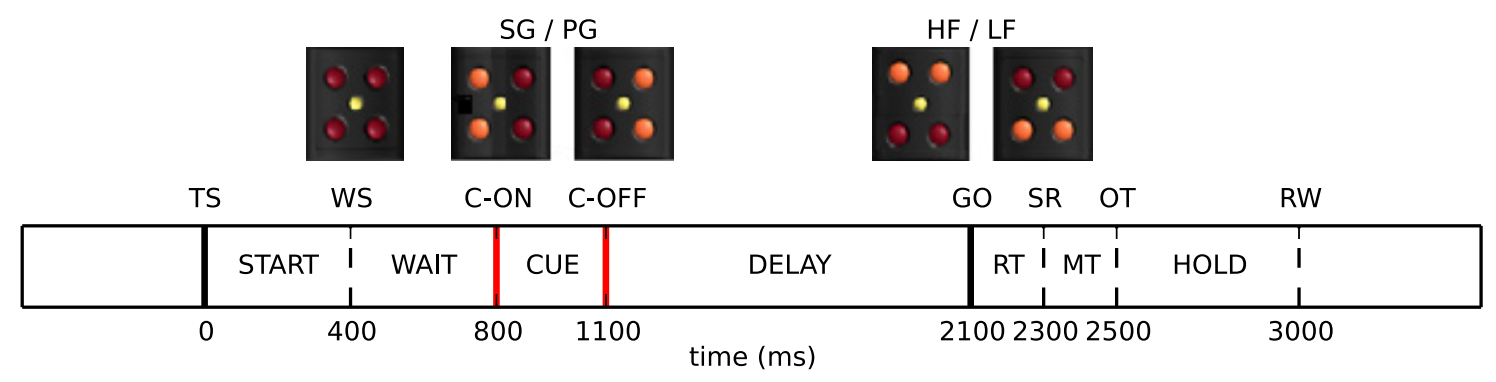

Figure 1. Reach-to-grasp experimental protocol. The trial start (TS) was self-initiated by the monkey by closing a home switch. A WS prepared the monkey for the visual cue (C+ until $\mathrm{C}-$ ) providing instruction about the grip type to use: SG or PG. One second later, a second visual cue turned on (GO), specifying the force needed to pull the object (HF or LF) and requesting movement initiation. The movement onset is marked by the switch release (SR). After object touch (OT), the monkey pulled the object and held it for $500 \mathrm{~ms}$ in a narrow position window until it was rewarded (RW). The timing of the behavioral events SR, OT, and RW, which follow the G0 signal, varied depending on the monkey's reaction time and movement speed.

havior (Vaadia et al., 1995; Riehle et al., 1997; Hatsopoulos et al., 1998; Jackson et al., 2003). However, the limited size of the data used in these studies, often comprising just a few simultaneously recorded neurons, raises a subsampling issue that makes these findings difficult to interpret and to generalize to larger populations of neurons.

Recent advances in electrophysiological technology provide the possibility to record hundreds of neurons simultaneously (Buzsáki, 2004; Schwarz et al., 2014). The high dimensionality of these data raises severe computational and statistical (multiple testing) problems due to the explosion of the number of possible synchronous spike patterns to be analyzed for significance. We recently developed a method that overcomes these issues by exploiting frequent item set mining to count pattern occurrences efficiently (Picado-Muiño et al., 2013) and by assessing pattern significance on the basis of a hierarchy of statistical tests (Torre et al., 2013). Borgelt and Picado-Muiño (2014) introduced a modification of this method to define synchrony in continuous time rather than by time binning, which outperforms the latter when synchrony is jittered by a few milliseconds. This approach was embedded in the data mining and statistical framework of the synchronous pattern detection and evaluation (SPADE) analysis for an efficient and statistically effective pattern detection.

In this study, we used the time-continuous SPADE analysis on simultaneous recordings from two monkeys engaged in an instructed-delay reach-to-grasp task to determine the occurrence of significant synchronous spike patterns in relation to behavior. Single-neuron spiking activity was recorded using a 100electrode Utah array chronically implanted in motor cortex. The task involved two grip modalities (side grip or precision grip) and two force levels (high or low) (Riehle et al., 2013). To perform a time-resolved analysis, we defined six successive temporal windows, or epochs, within each trial, from start until reward. We analyzed each epoch and trial type separately. Our analysis revealed a multitude of significant synchronous spike patterns, emerging more frequently during movement execution than during the instructed delay. The neuronal composition of patterns was specific to the grip modality, but only weakly related to the force level required. Patterns partially overlapped in neuronal composition. Clusters of overlapping patterns did not exhibit high specificity, suggesting that similar but not identical cell assemblies were active during different behaviors and that one neuron could participate in multiple assemblies.

Finally, neurons involved in significant patterns were aligned along a preferential mediolateral orientation in both monkeys. The neuronal composition of significant patterns was highly specific to the behavioral epoch and grip modality, indicating that different behaviors were related to the synchronization of differ- ent groups of neurons, which we interpret as the activation of different cell assemblies. These findings were consistent across the two monkeys and provide new insights on the functional role of spike synchronization in motor cortex.

\section{Materials and Methods}

Data

Experimental protocol and experimental setup. We analyzed electrophysiological data recorded from the motor cortex of two monkeys (Macaca mulatta), Monkey L (female) and Monkey N (male), during repeated execution of an instructed-delay reach-to-grasp task. The experimental protocol is described in Riehle et al. (2013). In summary, the monkey used one of two grips, either a full-hand side grip (SG) or a two-fingers precision grip (PG), to grasp and pull a cubic object along the horizontal axis. The object load was computer-controlled and was either $1 \mathrm{~N}$ for low force (LF) or $2 \mathrm{~N}$ for high force (HF) trials, respectively. The monkey had to hold the object in a narrow position window for $500 \mathrm{~ms}$ to obtain a food reward (drop of apple juice). Task instructions were provided by five LEDs (four red LEDs placed at the corners of a square and a yellow LED in the center) located just above the object. The task is illustrated in Figure 1. The monkey had to close a switch located at waist level to self-initiate the trial. After $400 \mathrm{~ms}$ from trial start, the yellow LED was illuminated as a warning signal (WS) for the cue occurring $400 \mathrm{~ms}$ later. The cue (illumination of the two right or the two left LEDs) was presented for $300 \mathrm{~ms}$ and instructed the monkey about the type of grip to be used to grasp the object. After a preparatory delay of $1000 \mathrm{~ms}$, a second cue (illumination of the two top or the two bottom LEDs) indicated the object load and also represented the GO signal instructing the monkey to execute the movement. Therefore, the monkey could perform one of four possible trial types: SGHF, SGLF, PGHF, and PGLF. The four trial types were presented in random succession within a recording session lasting for $\sim 15 \mathrm{~min}$, which consisted of a sequence of $\sim 130$ successful trial executions.

Both monkeys were chronically implanted in motor cortex with a 100 -electrode Utah array (Blackrock Microsystems) covering part of the dorsal premotor (PMd) and primary motor (M1) cortex (see Fig. $6 \mathrm{~A}$ for the array locations). The length of the electrodes was $1.5 \mathrm{~mm}$, with an interelectrode distance of $400 \mu \mathrm{m}$. Data were recorded using the 128-channel Cerebus acquisition system (Blackrock Microsystems). The signal from each active electrode (96 of the 100 electrodes were connected) was preprocessed by a head stage with unity gain and then amplified with a gain of 5000 . The signal was sampled at $30 \mathrm{kHz}$ ( 1 data point every $1 / 30 \mathrm{~ms}$ ) and filtered in two different frequency bands to be split into local field potentials (LFP, $0.3-250 \mathrm{~Hz}$ ) and spiking activity $(0.5-7.5 \mathrm{kHz}$ in Monkey $\mathrm{L}$ and $0.25-7.5 \mathrm{kHz}$ in Monkey N). The potential spike times were identified online on every channel by a threshold-crossing criterion and the corresponding waveforms saved in the Blackrock Central Suite as snippets of $1.6 \mathrm{~ms}$ (10 data points before the time of threshold crossing and 38 data points after) in Monkey L and $1.3 \mathrm{~ms}$ ( 10 data points before threshold crossing and 28 data points after) in Monkey $\mathrm{N}$ around the spike time. The threshold for spike selection was set online by the experimenter 
separately on every channel at the beginning of each recording day and controlled (and if necessary reset) at the beginning of each session. All behavioral data, such as stimuli, switch release, force traces for thumb and index fingers, and object displacement, were fed into the Cerebus system, sampled at $1 \mathrm{kHz}$, and stored for offline analysis.

Data preprocessing. The waveforms of the potential spikes were sorted offline separately on each electrode using the Offline Spike Sorter (version 3.3, Plexon) into the single-unit spiking activity (SUA) as described in Riehle et al. (2013). A preliminary analysis of the data revealed the presence of synchronous spikes across multiple SUAs at the sampling resolution of the recording system $\delta=1 / 30 \mathrm{~ms}$, a phenomenon often invisible to the bare visual inspection of the population raster plots. These spikes survived both the online and automatic artifact-rejection procedure provided by the Blackrock system and the offline spike-sorting procedure detailed in Riehle et al. (2013). The number of SUAs involved in these hypersynchronous events ranged from 2 to $>30$. A statistical analysis showed that the frequency of their occurrence largely exceeded the value expected under the observed population firing rate. Furthermore, fine-scale temporal correlation in the activity of the recorded neurons cannot explain these hypersynchronous events because it is not expected at submillisecond precision. Assuming that these events, the presence of which heavily affects any analysis of spike synchronization, are artifacts of unknown origin, we adopted a conservative approach and removed all of them from the data, which we then analyzed for synchronous spike patterns. This may have resulted in the removal of real synchronous spikes, which may lead to false-negative results. However, we privileged this compromise over the risk to have false-positive (FP) pattern detections. A time histogram of the population spiking activity triggered around the occurrence times of all hypersynchronous events and calculated using bins of width $\delta=1 / 30 \mathrm{~ms}$ revealed an increased spike count in the bins immediately preceding or after those events compared with other bins. Therefore, we additionally removed all spikes, even if isolated, occurring within a time interval $\delta=1 / 30 \mathrm{~ms}$ before or after any hypersynchronous event because they may as well reflect noise. For the analysis of spike patterns, we selected only SUAs with waveforms of high spike-sorting quality; that is, with a signal-to-noise ratio $>2.5$, as suggested by Hatsopoulos et al. (2004).

\section{SPADE analysis of spike synchrony}

We used the SPADE analysis (Picado-Muiño et al., 2013; Torre et al., 2013; Borgelt and Picado-Muiño, 2014) to search in massively parallel spike trains for patterns of synchronous spikes occurring more often than expected by chance under the null hypothesis $H_{0}$ of independence. SPADE overcomes the severe computational problem of counting pattern occurrences in massively parallel data characterized by an exponential explosion of the number of existing patterns and the consequent multiple testing problem of assessing the statistical significance of each such pattern.

Occurrence count of non-trivial patterns. SPADE defines synchronous spike patterns either by discretizing the time into bins of a given width $w$ and by considering spikes falling into the same time bin as synchronous (Picado-Muiño et al., 2013; Torre et al., 2013) or in continuous time by centering a window of width $w$ around each spike and collecting the spikes of all neurons falling inside that window (Borgelt and PicadoMuiño, 2014). We used the time-continuous version, which more reliably finds spike patterns with synchrony characterized by a small temporal jitter, using a window of width $w=3 \mathrm{~ms}$. The total number of synchronous patterns that occur in massively parallel spike train data is large (up to several millions), so counting the occurrences of each of these patterns by brute force algorithms is computationally not feasible. However, the large majority of these patterns occur only once; that is, they are "infrequent." Infrequent patterns can be discarded because either they would not be statistically significant after performing a statistical test or because their single repetition could not be associated with repeated behavior, as in the data we aim to analyze. Of the frequent patterns, i.e., the patterns that repeat at least two times, it is possible to discard all of those that repeat only as subsets of a larger pattern; that is, those that are "not closed." SPADE exploits a frequent item set mining algorithm (FPgrowth; Han et al., 2004) to restrict the search for patterns to those that are frequent and closed. This approach greatly speeds up the search for patterns and the counting of their occurrences. Details can be found in Torre et al. (2013).

Significance of pattern signature. Closed frequent patterns are evaluated for the statistical significance of their size $z$ (the number of spikes contained in the pattern) and their number of occurrences $c$, that is, of their signature $\langle z, c\rangle$. The probability of having patterns with signature $<z$, $c>$ under $H_{0}$ is evaluated for any signature $\langle z, c>$ using surrogate data. The surrogates are generated from the original data in such a way that correlations are destroyed while other features of the data are preserved to the largest extent possible. Among the many surrogate generation techniques known in the literature (see, e.g., Grün, 2009; Louis et al., 2010), we opted for spike dithering, which well preserves the firing rate profiles of individual units. Spike dithering generates surrogate data by displacing each individual spike around its original occurrence time by a random amount up to $\pm \delta_{j}$. We set $\delta_{j}=25 \mathrm{~ms}$, which is sufficiently larger than the synchrony precision $w$ to destroy synchronous patterns in the original data (Pipa et al., 2008) while preserving the rate profiles. The $p$-value of each signature $\langle z, c\rangle$ is then determined based on $K$ surrogate datasets (here, $K=10000$ ) as the fraction of surrogates that contain patterns with that signature. Signatures of patterns in the original data with a $p$-value that is lower than a significance threshold $\alpha$ are classified as statistically significant. We set $\alpha=0.01$ and applied the false discovery rate correction (Benjamini and Hochberg, 1995) for the number of different signatures-typically a few dozens-found in all surrogates. Because the surrogate data have the same firing rate profiles as the original data, these features are incorporated properly into the null hypothesis. Data with higher firing rates yield decreased statistical significance for pattern signatures compared with data characterized by lower rates. Indeed, the same pattern signature will be more likely to occur in surrogates obtained from higher-rate data due to the increased probability of chance synchronization (see Fig. 4 in Torre et al., 2013). Therefore, the evaluation of statistical significance is more conservative when applied on data of higher firing rates. Because the surrogates capture rate changes, the method is robust to sudden, simultaneous jumps in the neuronal firing rates (see Fig. 8 in Torre et al., 2013), which is a more extreme (and therefore more error-prone) version of the comodulation of firing rates observed in the data that we analyze here.

Pattern significance. In Torre et al. (2013), we showed on synthetic data that the presence of a real pattern $A$ tends to increase the size or the occurrence count (and thus the signature significance) of patterns that partially overlap with $A$. Pattern spectrum filtering is prone to classifying these patterns erroneously as significant themselves. To eliminate these FPs, we developed in Torre et al. (2013) a procedure named pattern set reduction, which evaluates the conditional significance of each pattern detected by pattern spectrum filtering given the occurrences of any other pattern overlapping with it and also returned as significant by pattern spectrum filtering. Borgelt and Picado-Muiño (2014) introduced a version of pattern set reduction compatible with the definition of synchrony in continuous time. For both the discrete and the continuous versions of SPADE, several variants of pattern set reduction exist (Torre et al., 2013). Among them, we opted for combined filtering, which tests for pattern significance given both pattern's supersets (superset filtering) and subsets (subset filtering) and retains only mutually significant patterns. Given two closed patterns $A$ and $B$ with signatures $\left\langle z_{A}, c_{A}>\right.$ and $\left\langle z_{B}, c_{B}>\right.$ such that $B \subset A$ (so that $z_{B}<z_{A}$ and $c_{B}>c_{A}$ ), combined filtering assesses the conditional significance of $B$ given $A$ and of $A$ given $B$. $A$ is considered significant given $B$ if the signature $\left\langle z_{A}-z_{B}+k, c_{A}\right\rangle$ is significant ( $k=2$ here). $B$ is considered significant given $A$ if the signature $\left\langle z_{B}, c_{B}-c_{A}+h\right\rangle$ is significant ( $h=1$ here). Each pattern that is still significant given the other is retained. If neither is, given that both have significant signatures and therefore at least one should be a true pattern, the pattern with the largest $z \cdot c$ score, that is, covering the largest number of spikes, is kept.

\section{Pattern specificity to behavior}

Our data consisted of parallel spike trains recorded during four different trial types (resulting from the combination of two grip modalities and two force levels). To associate the occurrence of significant patterns to behavior, we split each trial into six temporal epochs of $500 \mathrm{~ms}$ each (see 
Results) and searched for significant patterns separately in each combination of epoch and trial type. This resulted in 24 datasets analyzed for each session (one per epoch and trial type). We additionally defined four behavioral contexts: trial epoch, trial type, grip modality, and force level, each having multiple instances (six epochs, four trial types, two grip modalities, and two force levels, respectively). To determine whether specific ensembles of neurons were active in the different behavioral contexts, we investigated whether the significant patterns found during each instance of a behavioral context (e.g., during the start epoch) occur exclusively during that instance or whether instead they also occur during other instances of the same behavioral context (e.g., during other epochs).

Formally, given a context with $n$ instances let $\mathcal{P}_{i}$ be the ensemble of significant patterns found by SPADE during the $i$-th instance, $i=1, \ldots$, $n$. (For example, let $\mathcal{P}_{i}, i=1,2, \ldots, 6$ be the ensembles of patterns found during each of the 6 epochs). For each instance containing at least one significant pattern (i.e., $\mathcal{P}_{i} \neq \varnothing$ ), we defined its pattern specificity as the fraction of patterns in $\mathcal{P}_{i}$ that do not occur in any other $\mathcal{P}_{j}, j \neq I$, as follows:

$$
S_{i}=\frac{\left|\mathcal{P}_{i} \backslash\left(\cup_{j \neq i} \mathcal{P}_{j}\right)\right|}{\left|\mathcal{P}_{i}\right|}
$$

(for example, the fraction of patterns found during the start epoch that do not occur in other epochs). $S_{i}$ takes values in the interval $[0,1]$. It takes value 1 (or: 0 ) if all patterns found in the associated instance do not occur in any (or occur in at least one) other instance of the same context.

\section{Pattern clustering}

Clustering $\mathrm{n}$ patterns into $\mathrm{k}$ clusters. Patterns found as significant by the SPADE analysis may overlap in their neuronal composition. We characterized their similarity by performing a clustering analysis based on their neuronal composition. We first defined the distance $d(A, B)$ between any two patterns $A$ and $B$ as their Ochiai distance (Ochiai, 1957) as follows:

$$
d(A, B)=1-|A \cap B| / \sqrt{|A| \cdot|B|},
$$

which takes values between 0 (identical sets) and 1 (disjoint sets). We then employed the matrix $D$ of distances $d(A, B)$ between any two patterns as the metric in a classical $k$-medoids clustering algorithm (Kaufman and Rousseeuw, 1987). By fixing the number $k$ of clusters into which $n$ patterns have to be grouped, the algorithm selects $k$ points (here, patterns) as possible cluster centers (medoids) and builds clusters $C_{j}, j=$ $1, \ldots, k$, by assigning patterns to their closest medoid. The algorithm then computes the distance $d_{i}^{*}$ of each pattern from its closest medoid according to the metric $D$. The overall sum $\sum_{i=1}^{n} d_{1}^{*}$ gives an indication of the total "dispersion" of patterns around their closest medoid. The algorithm searches for the combination of $k$ medoids yielding the lowest dispersion $\Delta_{k}=\sum_{i} d_{1}^{*}$, and returns the associated configuration of clusters as the optimal $k$-clusters configuration $\mathcal{C}_{k}$ (having optimal dispersion $\Delta_{k}$ ). Given the discrete nature of the elements to be clustered (sets of points), a $k$-medoids algorithm is more suitable than algorithms computing averages of the elements, such as classical $k$-means clustering techniques.

Determining the optimal number $k$ of clusters. $\Delta_{k}$ is a non-increasing function of $k$ : a configuration with $k+1$ clusters and having a dispersion lower than or equal to $\Delta_{k}$ always exists, as long as $k<n$. A trivial example consists in considering a cluster with at least two patterns in the $k$-clusters configuration and removing from it one non-medoid pattern into a new isolated cluster. This operation removes an addendum from the dispersion of the first cluster and creates a 1-pattern cluster which has necessarily a dispersion equal to 0 , yielding an overall lower dispersion. To quantify the optimal number of clusters for a set of $n$ patterns, we thus penalized the total dispersion of a configuration by the number of its clusters, defining its cost by $R_{k}=\Delta_{k}+\rho \cdot k, k=1, \ldots, n$. We then determined the optimal number of clusters as $k^{*}=\arg \min _{k}\left\{R_{k}\right\}$ and grouped the patterns into the configuration $\mathcal{C}_{k *}$. A configuration $\mathcal{C}_{k+1}$ has a lower cost than $\mathcal{C}_{k}$ and is therefore to be preferred only if $R_{k+1} \leq R_{k}$ (or equivalently, if $\Delta_{k+1}+\rho \leq \Delta_{k}$ ); that is, if adding a cluster makes the cost drop by an amount that is not smaller than the
Table 1. Definition of trial epochs

\begin{tabular}{llcc}
\hline Epoch name & Trigger & $t_{\text {pre }}(\mathrm{ms})$ & $t_{\text {post }}(\mathrm{ms})$ \\
\hline Start & WS & 250 & 250 \\
Cue & $\mathrm{C}+$ & 250 & 250 \\
Early delay & $\mathrm{C}$ & 0 & 500 \\
Late delay & GO & 500 & 0 \\
Movement & SR & 200 & 300 \\
Hold & RW & 500 & 0 \\
\hline
\end{tabular}

The table summarizes the six different epochs defined for the analysis. Each epoch is a $500 \mathrm{~ms}$ time window starting at time $t_{\text {pre }}$ before a trigger and ending at time $t_{\text {post }}$ ffter that trigger $\left(t_{\text {pre }}+t_{\text {post }}=500 \mathrm{~ms}\right)$.

penalty coefficient $\rho$. We set $\rho=1$, which also represents the maximum distance between two patterns. This choice ensures that any two patterns in the same cluster share at least one neuron id. Indeed, whenever a cluster $C_{j}$ in the configuration $\mathcal{C}_{k}$ contains a pattern $A$ disjoint from all other patterns in $C_{j}$ (and thus contributing a +1 to the dispersion of $C_{j}$ ), there will exist at least one configuration of $k+1$ clusters having a lower cost than $\mathcal{C}_{k}$ : the one in which $A$ is an individual cluster separated from $C_{j}$.

Specificity of pattern clusters to behavior. Analogously to what was done for individual patterns, we defined the specificity of the ensemble $\mathcal{Q}_{i}$ of clusters found during the instance $i$ (e.g., epoch movement) of a behavioral context (e.g., epochs) as the fraction of clusters in $\mathcal{Q}_{i}$ which occurred only in the instance $i$, and in no other instance $j \neq i$ (e.g., other epochs), by the following:

$$
S_{i}=\frac{\left|\mathcal{Q}_{i} \backslash\left(\cup_{j \neq i} \mathcal{Q}_{j}\right)\right|}{\left|\mathcal{Q}_{i}\right|} .
$$

As well as pattern specificity, cluster specificity varies between 0 and 1 (all/no clusters found in the behavioral instance also occurred in other instances of the same behavioral context, respectively).

\section{Results}

\section{Pattern significance across epochs and trial types}

We analyzed spike data from multiple single neurons (up to $>100$ ) recorded simultaneously during an instructed-delay reach-to-grasp task (see Materials and Methods, Data) with SPADE (see Materials and Methods, SPADE analysis of spike synchrony) to detect statistically significant patterns of synchronous spikes. Patterns were significant if they repeated more frequently than expected given the firing rates of individual neurons under the null hypothesis of independence. For each monkey, we sorted one session per day, which was typically the first session of the day because there the monkey worked best (performed the largest number of correct trials). This resulted in 10 sessions from 10 different recording days. Each session lasted for $\sim 15 \mathrm{~min}$ and contained trials of four different types presented in random succession: SGHF, SGLF, PGHF, PGLF (see Materials and Methods, Data for more details on the experimental protocol). Within each behavioral trial, we identified six time windows, or epochs, of 500 ms duration each, related to specific triggers and thus behavioral contexts. Each epoch starts at time $t_{\text {pre }}$ before a specific trigger and ends at time $t_{\text {post }}$ after the trigger, as summarized in Table 1 and illustrated in Figure 2. Epoch start is centered around the appearance of the WS during a waiting period before the presentation of the visual cue. Epoch cue covers the time around the appearance of the visual cue providing prior information about the grip type. Epoch early delay captures the first half of the preparatory period after the offset of the visual cue, whereas epoch late delay covers the second half of this preparatory period until the presentation of the GO signal. Epoch movement is centered around the movement onset indicated by the switch release. Finally, epoch hold covers the $500 \mathrm{~ms}$ time period when the monkey holds the object in a fixed position to get the reward. Each epoch thus reflects a moment during the trial when different 


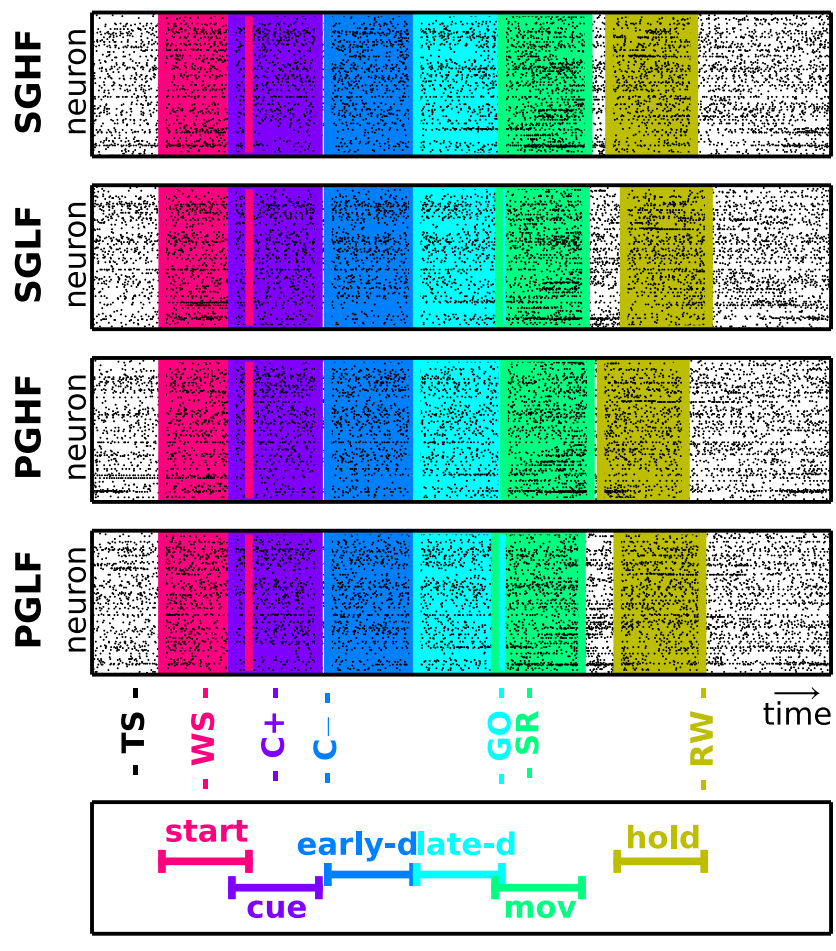

Figure 2. Trial types and epochs. Each panel shows the simultaneous spiking activity of all neurons (vertical axis) over time (horizontal axis) for four example trials (one per panel) of different types from a representative session in Monkey N. Each dot indicates one spike. The trials are aligned to trial start (TS). The six colored windows represent the position of the six epochs in the trials. The trigger associated to each epoch and the corresponding epoch name are shown at the bottom (for details, see Table 1). The movement (green) and hold (yellow) epochs are centered around triggers with occurrence times that changed from trial to trial depending on the reaction of the monkey to the $\mathrm{GO}$ signal and the movement time.

cognitive processes are supposed to take place: paying attention while waiting for the cue (start), receiving and/or processing instructions (cue, early delay, movement), preparing the correct grip and waiting for further instruction about the force level (early delay, late delay), performing the correct grasp (movement), and keeping the position of the object until reward (hold). Figure 2 illustrates these epochs as colored windows during one selected trial for each of the four trials types, along with the spike times recorded in parallel from all single neurons during the respective trials (black dots). As apparent from the figure, some epochs partially overlapped (e.g., start and cue or movement and hold). For epochs after the GO signal, this overlap varied from trial to trial with respect to the reaction and movement time of the animal.

With the goal of relating synchronous spike patterns to behavior, we separated each session into 24 different datasets corresponding to each combination of trial type and epoch. Specifically, each such dataset was obtained by concatenating all segments of spike trains corresponding to the same epoch (e.g., start) across all trials of the same type (e.g., SGHF) separately for each neuron. On average, a single session contained $\sim 130$ trials,; that is, $>30$ trials of each type. For each session, the individual datasets (e.g., SGHF start) were concatenated and then analyzed separately with SPADE for significant patterns of synchronous spikes of any size (i.e., number of neurons involved) and neuronal composition. A single session contained, on average, recordings from $70 \pm 13.76$ single neurons in Monkey L and $142.6 \pm$ 14.6 single neurons in Monkey N, resulting in millions of different possible patterns that SPADE reduced to a small number of significant patterns. The outcome of the analysis was a collection of 24 sets of significant patterns per session, one per trial type and epoch.

Figure 3 shows the results of a representative session from Monkey N. Figure $3 A$ illustrates the composition of neuron identities (horizontal axis) of each significant pattern in this session. Six patterns were found by SPADE, marked with labels 0 to 5 (vertical axis). Figure $3 B$ shows for each pattern the $p$-value (color coded) of the pattern signature in each epoch and trial type (logarithmic scale). White color corresponds to the significance threshold $\alpha=0.01$ for pattern signatures before application of the false discovery rate correction (signatures with a lower $p$-value may eventually still not be significant due to the statistical correction procedure). Squares with a black dot indicate the trial types and epochs in which the pattern was classified as statistically significant after correction.

\section{Pattern statistics across sessions \\ Pattern size and occurrences}

We performed the analysis outlined above separately for each session. Figure $4 A$ shows the total number of significant patterns found in the different epochs (horizontal axis; colors mark the contribution of each trial type) summed across the 10 sessions analyzed for each monkey. The highest number of significant patterns occurred during movement in both monkeys, but all epochs contained significant patterns. No relevant difference was observed between trial types. Figure $4, B$ and $C$, shows the average size and the average number of occurrences of the significant patterns detected during each epoch and trial type. Whiskers indicate the $\mathrm{SD}$, revealing a lower variability in pattern size across sessions compared with the number of pattern occurrences. Most patterns were composed of two neurons and repeated 100-300 times in each dataset. The largest pattern was found in PGLF trials during the early-delay epoch, comprising eight neurons and occurring twice. The remaining patterns had a size $\leq 3$ for Monkey L and $\leq 4$ for Monkey N. Typically, the number of occurrences needed for a pattern to become significant dropped exponentially with pattern size, as shown by Torre et al. (2013).

\section{Spatial arrangement of neurons involved in patterns}

The interelectrode distance on the recording array was 400 and $566 \mu \mathrm{m}$ in the horizontal/vertical and the diagonal direction, respectively. The vast majority of neurons forming significant patterns were recorded from electrodes located $0.4-2.4 \mathrm{~mm}$ apart (Fig. 4D), corresponding to a separation of $1-6$ electrodes on the array along the horizontal/vertical direction (Fig. 5A).

A number of experimental studies showed a decay of spike correlation among neurons placed at increasing distances (Kwan et al., 1987; Gray et al., 1989; Murthy and Fetz, 1996; Berger et al., 2007; Smith and Fetz, 2009). SPADE makes it possible to test such a relation on massively parallel spike train data. Here, we studied how the fraction of neuron pairs belonging to any significant pattern varies with the distance between the two neurons in the pair. To this end, we extracted from each pattern (of any size) found as significant in any session, epoch, and trial type all pairs of neurons composing the pattern, and calculated the distance between the neurons in each pair. Next, we built the histogram of the number of pairs recorded at a given distance $(0.4-5.4 \mathrm{~mm}$ in steps of $1 \mathrm{~mm}$ ) and, finally, we corrected the values taken by each bar in the histogram by the overall number of recorded neuron pairs at that distance, which varied with distance. The results are shown in Figure 5. For both monkeys, the fraction of pairs of neurons involved in the same pattern and recorded within a given 
Monkey N: session i140617-001
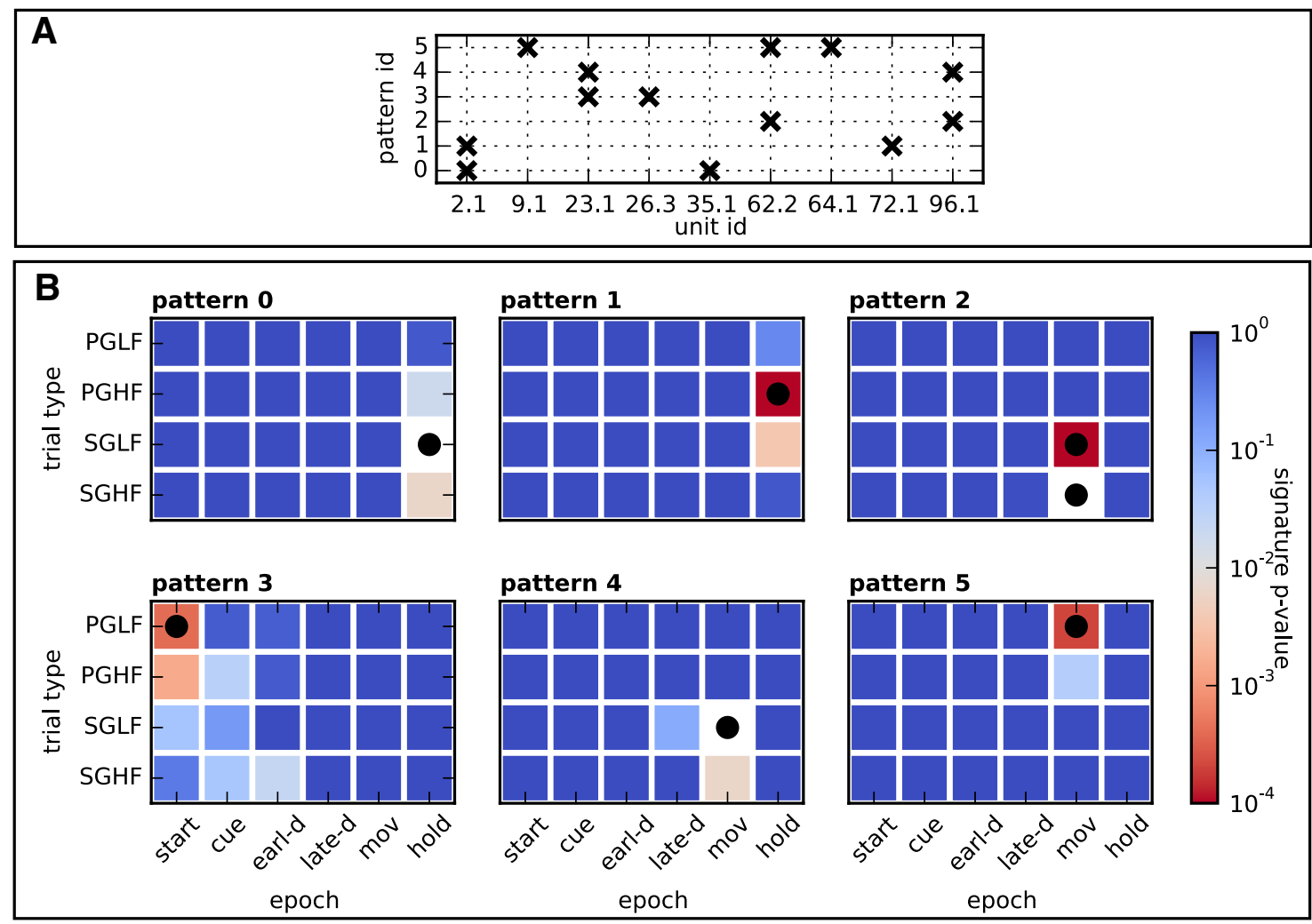

Figure 3. Significant patterns in one representative session. $\boldsymbol{A}$, Composition of neuron identities (horizontal axis) of each significant pattern in the session (vertical axis, pattern IDs 0 -5). $\boldsymbol{B}$, Each panel shows one color map associated to a specific significant pattern. The color map shows the uncorrected $p$-value of the pattern's signature in each trial type and epoch (color progression in logarithmic scale). Squares with the black dot indicate the session's trial type and epoch where the pattern was classified as statistically significant.

distance range, corrected by the total number of recorded neuron pairs placed at that distance, decayed with distance, in agreement with previous findings (Berger et al., 2007). The trend was stronger for Monkey $\mathrm{N}$ than for Monkey L, but was present in both animals. Summing across sessions, trial types, and epochs, the data from Monkey L contained 71 pairs of SUAs involved in the same pattern of 24,905 recorded pairs $(\approx 0.29 \%)$, whereas the data from Monkey N contained 101 of 101,204 correlated pairs $(\approx 0.1 \%)$. Note that these seemingly low numbers are still considerably higher than the effective significance threshold used in the analysis ( $1 \%$, corrected for false discovery rate over the number of pattern signatures found in the data, several hundreds, and further decreased by pattern set reduction, which removes chance overlapping patterns, leading to an effective significance level approximately equivalent to $0.01 \%$ or lower; see Materials and Methods, SPADE analysis of spike synchrony for details). The overall statistical analysis was very conservative compared with similar studies performed on recordings involving only a few simultaneously recorded neurons, which was necessary to avoid FPs.

Although up to four single units (but typically one or two) were recorded from individual electrodes, the significant patterns found never involved more than one SUA per electrode. This is partly due to the properties of the recording system we used to collect the data. To identify the spikes recorded at each electrode, data snippets of 1.6/1.3 ms duration in Monkey L/N, respectively, were centered around the time points of threshold crossing. Each snippet contained 10 data points (one every 1/30 ms) before and $38 / 28$ data points after in Monkey L/N, respectively. These wave- forms were then sorted offline. The spike times were set to the threshold crossing times. During each time window used to isolate a spike waveform, no subsequent overlapping waveforms could be detected on the same electrode. Therefore, these additional waveforms, if occurring, were missed, thereby leading to a missed detection of synchronous events. For this reason, although we defined synchrony by allowing for a maximum temporal jitter of $3 \mathrm{~ms}$, no synchronous events composed of neurons recorded on the same electrode occurred often enough to become statistically significant. As shown above, the probability for neurons to be involved in a synchronous spike pattern decayed with their distance. This suggests that many more patterns are likely to have occurred among the neurons close to the same electrode than those we detected.

To relate the spatial organization of synchrony to behavior, we additionally investigated the location on the array of neurons involved in synchrony. Figure $6 \mathrm{~A}$ shows, for Monkeys $\mathrm{L}$ and $\mathrm{N}$, the location of the array on motor cortex with respect to central sulcus, arcuate sulcus, and precentral dimple (compare Fig. 1 in Riehle et al., 2013). Figure $6 B$ shows the total number of SUAs recorded on each electrode summed over all sessions (color coded). In total, 700 SUAs were considered in Monkey L and 1426 in Monkey N. All selected sessions were recorded on separate days, typically with several days in between the recordings. Based on previous studies (Dickey et al., 2009; Vaidya et al., 2014), we expect the fraction of identical cells across sessions to be small. To verify this, we investigated whether patterns that occurred in more than one session resulted from identical underlying neurons. To this end, we identified patterns that were found 


\section{A}

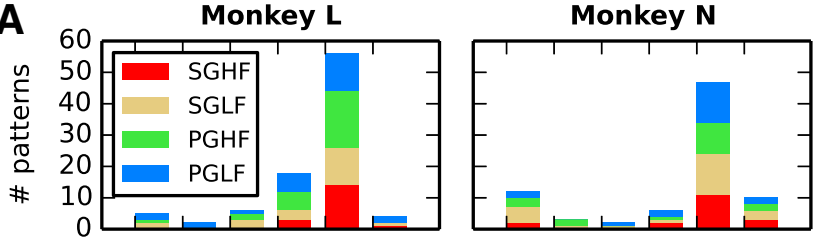

B

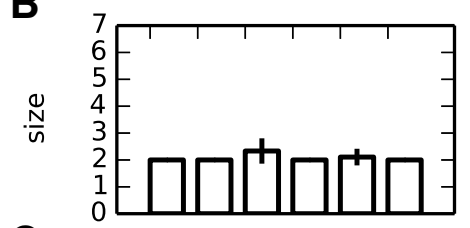

C

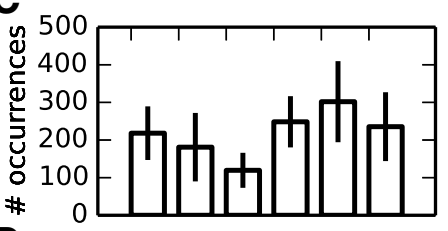

D

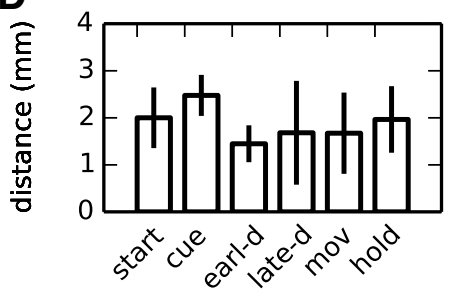

epoch
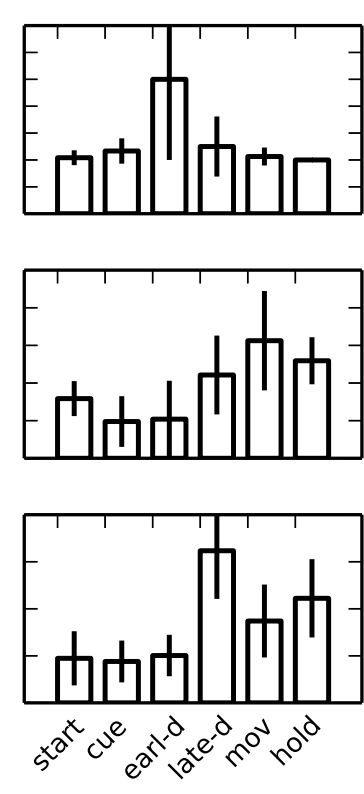

epoch

Figure 4. Pattern statistics across sessions. Various statistics of the significant patterns detected across the 10 selected sessions separately for each monkey (columns). $\boldsymbol{A}-\boldsymbol{C}$, Total number of significant patterns in each epoch (colors mark the contribution of each trial type; $A$ ), their average size $(\boldsymbol{B})$, and their average number of occurrences $(\boldsymbol{C})$. $\boldsymbol{D}$, Average distance between electrodes on which neurons are recorded that are involved in the same pattern. Whiskers in panels $\boldsymbol{B}-\boldsymbol{D}$ indicate the $S D$ when it is $>0$.

in more than one session on the same electrodes (regardless of the ID associated with each participating SUA in each session) and found only six such patterns in Monkey L and two in Monkey N. None of these patterns occurred in $>2$ sessions. The inspection of the SUAs recorded at these electrodes revealed, based on their waveforms, their interspike-interval histograms and their activity profiles obtained during each of the four trial types, that the two repetitions always consisted of different neurons. Figure $6 C$ shows the color-coded number of SUAs recorded on each electrode and involved in a significant pattern summed over sessions and normalized by the number of SUAs recorded on that electrode and shown in Figure 6B. Neurons involved in significant patterns did not exhibit any spatial arrangement specific to the trial type or to the epoch (data not shown). We also investigated the spatial orientation of pairs of neurons involved in synchronous spiking activity and computed their orientation on the grid as angles between $-\pi / 2$ and $+\pi / 2$, where 0 degrees correspond to the anterior-posterior orientation and $\pm \pi / 2$ to the mediolateral one. The pattern orientations are shown in Figure $6 C$ as overlaid black lines between any two electrode positions from which the two respective neurons were recorded. Visual inspection did not reveal a clear preferred orientation.

However, when building the orientation histogram in polar coordinates by pooling the angles of all pattern orientations found in 8 ranges of amplitude $\pi / 8$ each, and by normalizing each bar by the total number of recorded neuron pairs that could have

formed a pattern orientation in that range (Fig. 6), we found for both monkeys a preferred orientation of neuron pairs involved in significant patterns in the mediolateral orientation (see Fig. $6 E$ for patterns detected during the movement epoch). Indeed, the number of such pairs is significantly higher $(p<0.05$ in both monkeys) than expected on the basis of the fraction of recorded neuron pairs placed along that orientation. This orientation was shown previously to subserve the tight functional coupling between proximal and distal representations of the upper limb during the performance of complex reach-to-grasp movements (Riehle et al., 2013). We also explored whether the pattern orientation was specific to the trial types or the behavioral epochs, but we did not find any qualitative differences to the result found for the overall average (data not shown).

\section{Specificity of patterns to behavioral context}

To assess whether patterns are specific to behavior, we considered four behavioral contexts: epoch, trial type, grip modality and force level. Each behavioral context has multiple instances: six trial epochs, four trial types, two grip modalities, and two force levels, respectively. For each instance $i$, we quantified its pattern specificity $S_{i}$ by the fraction of patterns found in that instance (e.g., start epoch) and not in other instances of the same context (e.g., other epochs), as defined in Equation 1. $S_{i}=1$ implies maximal specificity and $S_{i}=0$ no specificity.

Starting with the six different epochs from start to hold, we determined separately for each session the ensemble $P_{i}$ of significant patterns found in that session during each epoch $i$, pooling across all trials regardless of the trial type. For instance, Figure $3 B$ shows that, in our representative session, the hold epoch contained 2 patterns, named with IDs 0 and 1 , and these patterns were only significant in this epoch, which yields pattern specificity 1. Similarly, the start epoch and the movement epoch contained 1 and 3 patterns, respectively, occurring in those epochs only and yielding again specificity 1 . The other epochs of this session contained no significant pattern and no pattern specificity value could be assigned to them. For each epoch, we then averaged its pattern specificity across sessions. The results, illustrated in Figure $7 A$ separately for each monkey, indicate that most epochs exhibited a high $(>0.6)$ or very high $(>0.8)$ pattern specificity; that is, they contained a majority of patterns that did not occur in other epochs. An exception is represented by the cue epoch in both monkeys and by the early-delay epoch in Monkey $\mathrm{N}$, which show specificity 0.5 . However, all 3 cases resulted from an average computed across 2 sessions only, taking values 0 and 1 , whereas the other sessions did not contain significant patterns in that epoch. Therefore, we could not draw a conclusion on pattern specificity for these epochs. In contrast, the late-delay, the movement, and the reward epochs exhibited pattern specificity 1 or close to 1 in both monkeys. Interpreting significant patterns as the signatures of cognitive processing that involves the activation of specific cell assemblies, these results suggest that assemblies become selectively active with respect to specific behavioral demands.

We analogously computed pattern specificity for each of the four trial types regardless of the epochs during which they were found. In our example from Figure $3 B$, the pattern specificity for the different trial types in the representative session was 0 for SGHF, 0.67 for SGLF, and 1 for PGHF and PGLF. The pattern specificity for the different trial types averaged across sessions, shown in Figure $7 B$, ranged between 0.15 (low) and 0.6 (high). Because each trial type was defined as the combination of the grip modality and the force level required to accomplish the task, we 

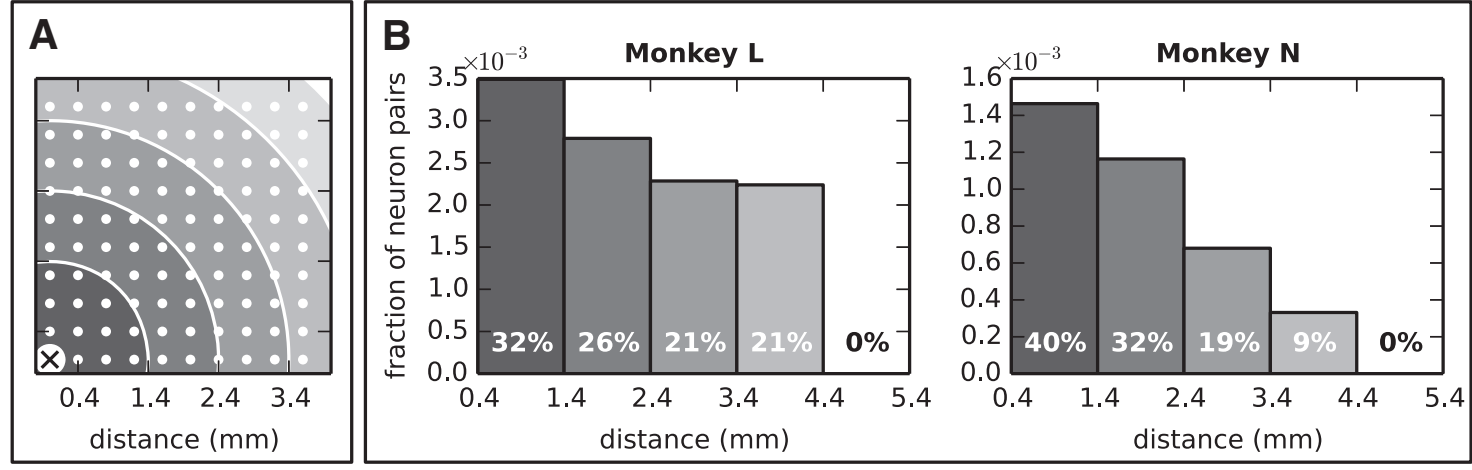

Figure 5. Dependence of synchrony on electrode distance. $A$, Distance of the electrodes on the recording array (white dots) from one reference electrode (cross at the bottom left corner). Dark to light shaded areas cover regions at progressively larger distances from the reference electrode from $0.4 \mathrm{~mm}$ up to $5.4 \mathrm{~mm}$ in steps of $1 \mathrm{~mm}$. $\boldsymbol{B}$, Histogram showing, for each distance range, the ratio between the number of neuron pairs involved in the same pattern and placed within that range from each other (summed across patterns found in any session, epoch and trial type) and the total number of recorded neuron pairs placed at that distance (vertical axis). The numbers inside each bar represent the relative height of each bar with respect to the sum of all bars; that is, the relative fraction of neuron pairs involved in the same patterns placed at a given distance.
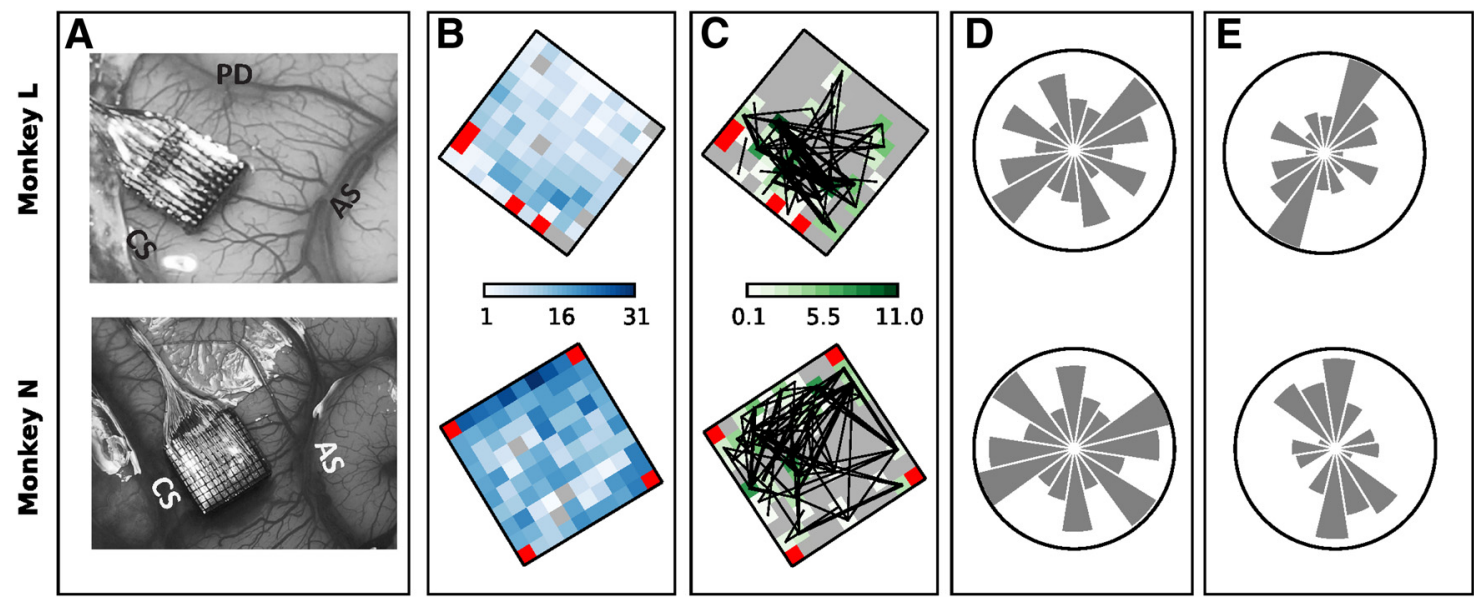

Figure 6. Spatial arrangement of neurons participating in significant patterns. A, Locations of the electrode arrays in the motor cortex in the right hemisphere of the two monkeys. CS, Central sulcus; $\mathrm{AS}$, arcuate sulcus; $\mathrm{PD}$, precentral dimple. $\boldsymbol{B}$, Color maps of the total number of $\mathrm{SUAs}$ recorded on each electrode of the recording array summed across all selected sessions. Red squares indicate the four unconnected electrodes. $\boldsymbol{C}$, Color maps showing, for each electrode, the total number of patterns found that involved neurons recorded on that electrode. The number is obtained separately for each session (summing across all epochs and trial types) divided by the total number of single neurons recorded on the electrode in the session and finally summed across sessions. The final value can exceed the number of sessions (10) if, for instance, in each session there were more significant patterns involving the electrode than neurons recorded by that electrode. Gray squares correspond to a value of 0 and indicate electrodes that never recorded single neurons involved in significant patterns. Black lines connect electrode pairs extracted from each significant pattern found. $D$, Number of all possible pairs of recorded neurons placed along each orientation. Each panel shows a bar chart in polar coordinates. The direction of each bar corresponds to one orientation on the recording array, whereas the length of the bar represents the total number of neuron pairs placed along that direction computed for each session and summed across sessions. The circles have a diameter of 5000 for Monkey L and of 18,000 for Monkey N. E, Spatial orientation of all pairs of neurons involved in significant patterns found during movement as a fraction over the total number of recorded neuron pairs with that orientation shown in $\boldsymbol{D}$.

disentangled the two aspects by further computing the pattern specificity for the grip modality (SG vs PG) regardless of the force level and the epoch (Fig. 7C) and for the force level (HF vs LF) regardless of the grip modality and the epoch (Fig. $7 D)$. The results show high or very high grip-type specificity $(>0.8)$ and low force-type specificity $(<0.5)$. The combination of both explains the moderate specificity values found for the trial types. Following the same line of argument as before, we concluded that, in our data, specific grip modalities, but not specific force levels, were associated with the activation of specific cell assemblies.

\section{Specificity of pattern clusters}

Individual neurons often participated in multiple significant patterns (Fig. 3A). When interpreting patterns as signatures of cell assemblies associated with a specific behavior or condition, different but overlapping patterns may reflect either a common cell assembly with an activation that recruits each time only a subset of its composing neurons, or different cell assemblies that share some of the composing neurons. To distinguish between these two hypotheses, we investigated whether different but overlapping patterns were found during the same behavioral context or not, which support the first and the second hypothesis, respectively. To this end, for each session, we first grouped by means of a clustering algorithm all patterns found during that session (across all trial types and epochs) into ensembles characterized by a similar neuronal composition (for details, see Materials and Methods, Pattern clustering). Given $n$ total patterns in a session, the clustering algorithm evaluates for each integer $k=1,2, \ldots, n$ how well these patterns can be separated into a configuration of $k$ clusters by assigning a cost to that configuration, as illustrated in Figure $8 B$. The algorithm selects the optimal number $k^{*}$ of clusters and the associated optimal configuration based on the lowest cost. 

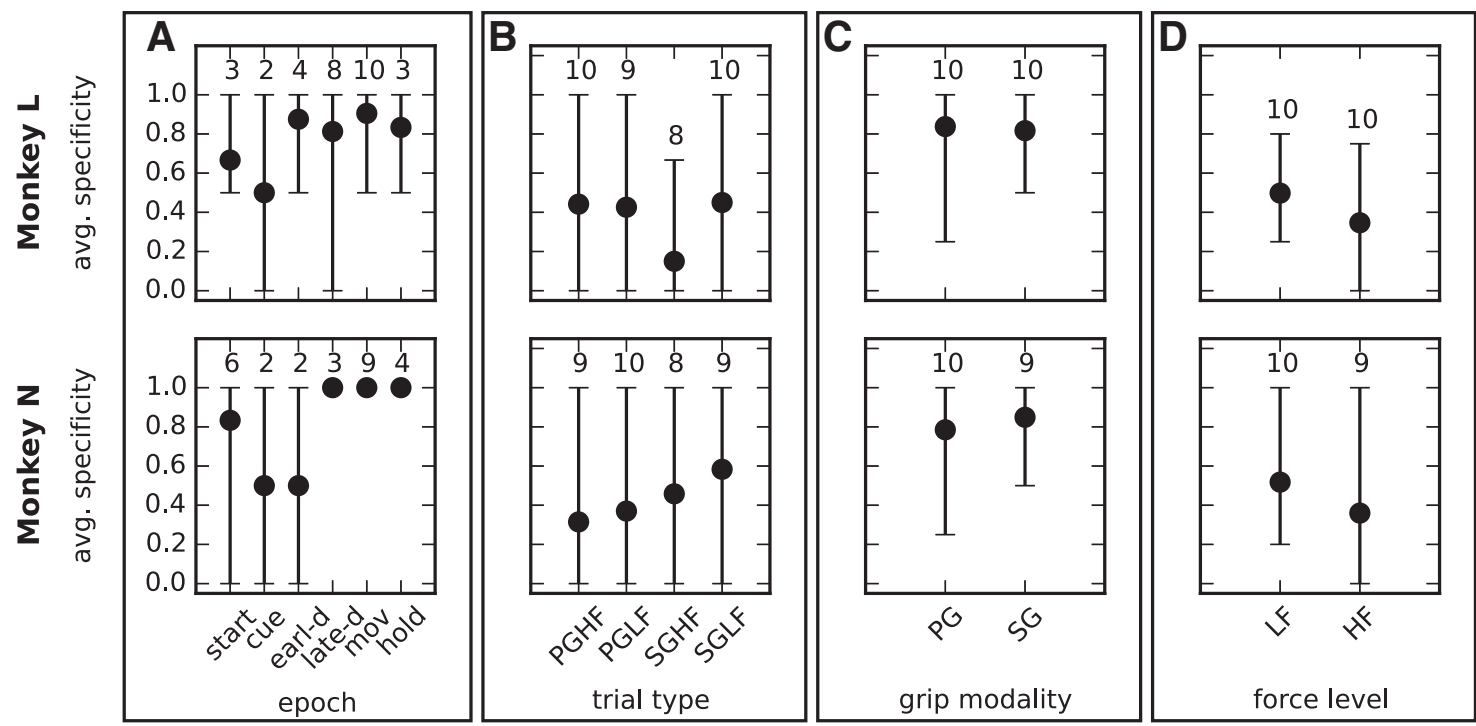

Figure 7. Pattern specificity for different behavioral contexts. The four panels show the pattern specificity at each instance of the four behavioral contexts: epochs $(\boldsymbol{A})$, trial types $(\boldsymbol{B})$, grip modalities $(\boldsymbol{C})$, and force levels $(\boldsymbol{D})$. The values were calculated for each monkey across all sessions. The bars extend between the minimum and maximum value across sessions. The numbers above each bar indicate the number of sessions that actually contained significant patterns in that instance (e.g., in that epoch). When this number is 2 , then the values taken by the 2 corresponding sessions are the ends of the range. The other sessions did not contain significant patterns, so a pattern specificity value could not be calculated.

A

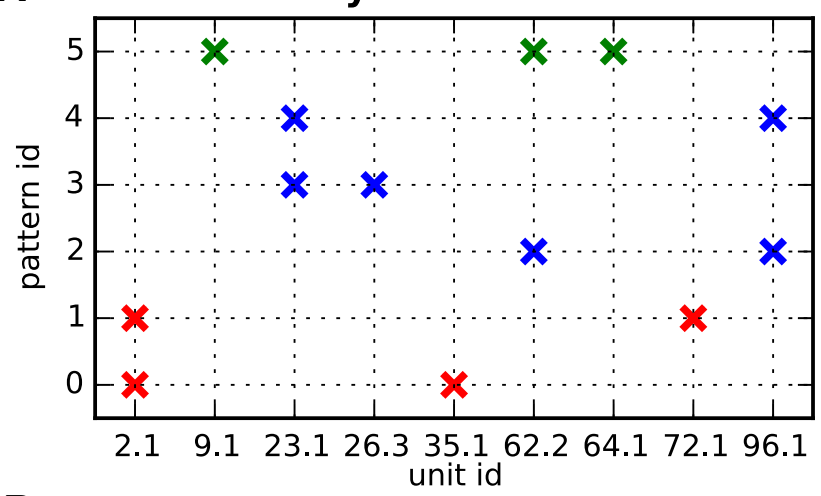

B

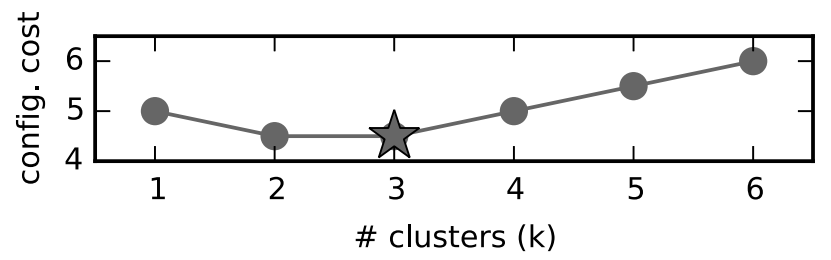

Figure 8. Outcome of clustering for one representative session. $A$, Crosses indicate the neuron IDs (horizontal axis) of each pattern detected in the session (vertical axis; as in Fig. 3A). Red, blue, and green colors identify the membership of each pattern to one of $k^{*}=3$ clusters. $B$, Cost associated with each number $k$ of clusters, $k=1,2, \ldots, 6$.

Figure $8 A$ illustrates, for the already discussed representative session of Monkey N (compare Fig. $3 A$ ), how its $n=6$ patterns are optimally clustered into $k^{*}=3$ clusters. Each cluster is identified by a different color. Figure $8 B$ shows the cost of each configuration of $k=1,2, \ldots, 6$ clusters with a minimum at $k^{\star}=3$.

Moreover, for each session we computed the cluster specificity of each behavioral context (Eq. 2), which was analogous to what was done for pattern specificity. For instance, the cluster specificity of one epoch reflects the fraction of clusters found in that epoch (regardless of the trial type) and in that epoch only. By definition of specificity, no values can be assigned to datasets containing no significant patterns and for which no cluster can therefore be identified.

We performed the pattern clustering and computed the cluster specificity of each behavioral context (epoch, trial type, grip modality, and force level) separately on each session. Figure 9 shows the results averaged across all sessions for each monkey. Epochs have an overall cluster specificity of 0.3 to 0.5 (Fig. 9A), which drops to $<0.2$ for trial types (Fig. 9B), grip modalities (Fig. 9C), and force levels (Fig. 9D). These results indicate that groups of similar patterns are not specific to any of the defined behavioral contexts, unlike individual patterns. This finding supports the second hypothesis outlined above, namely that different cell assemblies, as identified by different patterns, are associated with different behavioral contexts despite involving partially identical neurons.

\section{Discussion}

In this study, we examined the emergence of patterns of precise synchronous spiking activity in massively parallel recordings from macaque motor cortex during execution of an instructeddelay reach-to-grasp task (Riehle et al., 2013). We used the SPADE analysis (Torre et al., 2013), which combines a frequent item-set mining approach to find repeating patterns of synchronous spikes efficiently in large datasets with a hierarchy of statistical tests to determine their significance without incurring severe multiple testing issues.

We found significant patterns in both monkeys, occurring mainly, but not exclusively, during movement (Fig. 4A). In previous studies (Riehle et al., 1997; Kilavik et al., 2009; Denker et al., 2011), we found excess spike synchrony preferably during the preparatory period in epochs related to expected events. In our current study, all epochs were aligned to expected cues and, indeed, we found patterns during all of these epochs. Interestingly, we found more patterns in Monkey L before the GO signal compared with Monkey N, despite having recorded significantly less neurons in the first animal. Monkey L also exhibited shorter reaction times, which may be interpreted as a better anticipation of the GO signal and may explain the larger number of patterns 

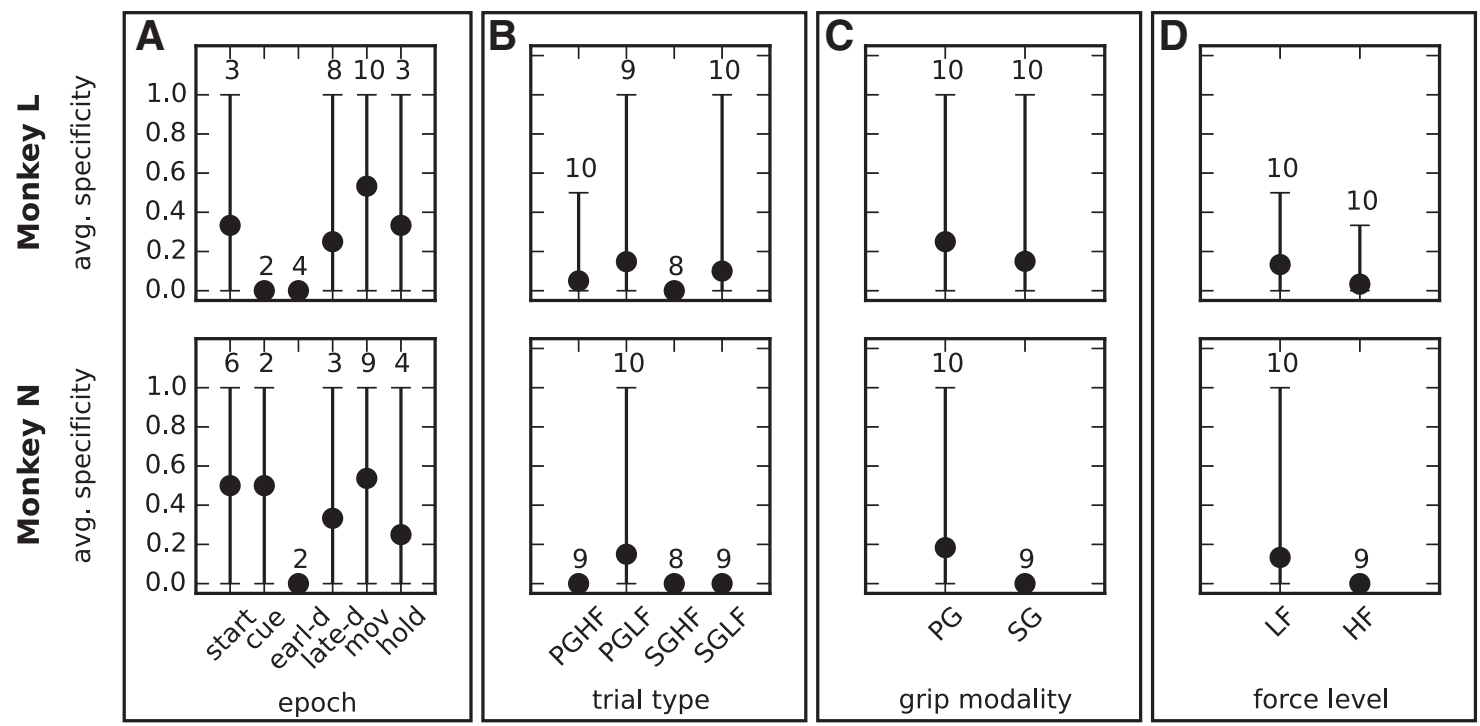

Figure 9. Cluster specificity for different behavioral contexts. The four panels show the cluster specificity value for epochs $(\boldsymbol{A})$, trial types $(\boldsymbol{B})$, grip modalities $(\boldsymbol{C})$, and force levels $(\boldsymbol{D})$ analogous to Figure 7 for pattern specificity.

found during the delay epochs. In addition, the movement task requested in the current experiment was more complex and required more concentration by the subjects compared with our former studies. This may explain the larger amount of patterns found during the movement period compared with the other epochs.

The found patterns involved primarily two, but also three or more neurons (Fig. 4B). These numbers were likely reduced by the fact that we preprocessed the data by removing all spikes forming hypersynchronous events at sampling precision $\delta=1 / 30 \mathrm{~ms}$, which we assumed to be artifacts because they occurred beyond the chance level determined by the firing rates and at a too high temporal precision to be explained by true correlations. By removing hypersynchronous spikes, we also likely removed real spikes from some of the synchronous events that we found.

Each significant pattern occurred up to hundreds of times (Fig. 4C). Even though the neurons forming one pattern typically belonged to non-neighboring electrodes (Fig. 4D), the probability that two neurons belonged to the same pattern decreased with their distance (Fig. 5B). Previous studies also reported spike synchrony decaying with distance within a similar (Berger et al., 2007) or shorter (Kwan et al., 1987; Gray et al., 1989; Murthy and Fetz, 1996; Smith and Fetz, 2009) spatial range.

We further determined whether patterns were specific to the behavioral context such as behavioral epoch, trial type, grip modality, and force level. Pattern specificity for a behavioral context was defined such to take a large value when a majority of the patterns were detected as significant only in that context (e.g., during the start epoch or during side grip trials). High pattern specificity for one behavioral context may indicate that the associated patterns are involved in information processing for that specific context. Our results revealed for both monkeys a high pattern specificity for epochs (in particular start, late-delay, movement, and hold; Fig. 7A) and grip modality (Fig. 7C), but not for force level (Fig. 7D). The high specificity for individual epochs matches earlier studies showing that distinct populations of neurons are involved in movement preparation and execution in instructed-delay motor tasks (Wise et al., 1983; Riehle and Requin, 1989; for review, see Riehle, 2005; Confais et al., 2012).
The present observation suggests that synchronous spiking activity may play a critical role in the processing of information in motor cortex. The contrasting degree of specificity between grip types and force levels is likely to reflect functional properties of the cortical location at which the array was implanted. In a previous study (Milekovic et al., 2015), we demonstrated that grip types could be precisely decoded from the neuronal data, whereas force-level decoding was less precise. It seems therefore plausible that synchronous spiking activity may contribute to the fine tuning of neuronal activity for different types of movements, for example, by engaging an assembly of neurons targeting similar sets of muscles (Jackson et al., 2003).

Some of the patterns found in the data partly shared the same neurons. We investigated whether overlapping patterns may be associated to a common cell assembly that required only a subset of its neurons to fire synchronously each time. Otherwise, the presence of overlapping patterns would suggest that the same neuron participates in different assemblies at different times. To discriminate between these two cases, we grouped similar patterns and computed the specificity of behavioral contexts in terms of pattern clusters rather than individual patterns. The results, shown in Figure 9, indicate a very low cluster specificity for all behavioral contexts, supporting the second hypothesis that overlapping patterns indeed reflect the activation of different assemblies.

We also investigated the spatial organization of significantly correlated pairs of neurons in the forelimb area of monkey motor cortex and found a preferential alignment of the patterns along the mediolateral axis. This orientation is perpendicular to the dominant orientation reported for traveling waves of beta oscillations in the local field potential (Rubino et al., 2006) or the orientation of information flow between individual neurons as revealed by Granger analysis (Takahashi et al., 2015). The mediolateral alignment is, however, consistent with the dominant alignment of synchronized pairs of neurons in the motor cortex along the central sulcus (Kwan et al., 1987). This alignment may subserve the tight functional coupling between proximal and distal representations of the upper limb during the performance of complex reach-to-grasp movements, as already shown in Riehle et al. (2013). In that study, we demonstrated that the first com- 
ponents of the LFP movement-related potential were predominantly related to proximal movements $(\mathrm{P} 1-\mathrm{N} 1$, reach), having a larger amplitude at a medial location on the motor cortical surface, whereas the subsequent components were predominantly related to distal movements ( $\mathrm{P} 2-\mathrm{N} 2$, grasp) with larger amplitudes at a lateral location. Therefore, the two perpendicular orientations in motor cortex may be a substrate of a parallel spatiotemporal organization of motor cortex for the coordination of upper limb movements. Neurons positioned along the mediolateral axis may be synchronously activated by traveling waves running along the rostrocaudal axis, thus inducing significant synchrony patterns between these two cortical representations. Further investigations will be required to confirm this interpretation.

Former analyses of parallel spike trains for excess spike synchrony using unitary events analysis revealed behavior-related spike synchrony between pairs or triplets of neurons (Riehle et al., 1997; Kilavik et al., 2009; Ito et al., 2011). Part of these findings were also reproduced by other methods (Pipa et al., 2007; Shimazaki et al., 2012). However, these studies were performed on simultaneous recordings of a small number of neurons only. Therefore, comparing our results with those obtained previously is difficult for two reasons: (1) we used different statistics and (2) we analyzed massively parallel spike trains. Concerning the former, SPADE tests the significance of each pattern by first evaluating the probability that any pattern of the same size (and not only the specific pattern) would occur the same or a larger number of times under independence. On large data, this approach performs better than direct tests, which incur multiple testing issues. A comparison of these methods on simulated and real data will be part of future work. Concerning the second, one may expect to observe synchrony between larger groups of neurons as the size of the observed population increases because the undersampling is reduced. However, reasoning based on combinatorial calculus shows that subsampling remains an obstacle to observe higher-order correlations. Let us assume for simplification that the cortical volume in which the $3.6 \times 3.6 \mathrm{~mm}$ arrays were inserted contained $M=1,000,000$ neurons (100,000 neurons/ $\mathrm{mm}^{3}$ ), $N \approx 100$ of which we randomly sampled; then, we can use the hypergeometric distribution to calculate the probability of sampling $\xi$ versus $\xi+1$ out of the $C$ neurons forming an assembly. From this calculation, we infer that the probability of sampling, in parallel recordings of $N=100$ neurons, $\xi=3$ cells belonging to an assembly of size $C=100$ would be $\sim 300$ times smaller than the probability of observing only $\xi=2$ of those neurons. Our choice of $C$ derives from theoretical considerations based on the synfire chain model (Abeles, 1991) and corresponds to the size of one group in the chain for stable synchrony propagation (Diesmann et al., 1999).

Conversely, the fact that we found only synchronous patterns of small size may speak for a generalized version of the synfire chain model, namely the synfire braid (Bienenstock, 1995) or polychrony (Izhikevich, 2006) model. This model allows for different spike transmission delays that are set such that spikes emitted from neurons in one group at different times arrive synchronously to the next group. One would then rather expect to observe primarily spatiotemporal patterns than spike synchrony. In light of these considerations, the few highly significant synchronous spike patterns of size 3-8 found in our data provide a strong indication that larger pools of synchronous cell assemblies may underlie cortical processing. Continuous progress in electrophysiological technology promises to enable in the near future simultaneous recordings of thousands of neurons
(Schwarz et al., 2014), which may be better suited to identifying larger portions of synchronous cell assemblies.

\section{References}

Abeles M (1982) Role of cortical neuron: integrator or coincidence detector? Isr J Med Sci 18:83-92. Medline

Abeles M (1991) Corticonics: neural circuits of the cerebral cortex. Cambridge: Cambridge University.

Azouz R, Gray CM (2000) Dynamic spike threshold reveals a mechanism for synaptic coincidence detection in cortical neurons in vivo. Proc Natl Acad Sci U S A 97:8110-8115. CrossRef Medline

Azouz R, Gray CM (2003) Adaptive coincidence detection and dynamic gain control in visual cortical neurons in vivo. Neuron 37:513-523. CrossRef Medline

Benjamini Y, Hochberg Y (1995) Controlling the false discovery rate: a practical and powerful approach to multiple testing. J R Stat Soc Ser B 57:289-300.

Berger D, Warren D, Normann R, Arieli A, Grün S (2007) Spatially organized spike correlation in cat visual cortex. Neurocomputing 70:21122116. CrossRef

Bienenstock E (1995) A model of neocortex. Network 6:179-224. CrossRef Borgelt C, Picado-Muiño D (2014) Simple pattern spectrum estimation for fast pattern filtering with coconad. In: Advances in intelligent data analysis XIII, Vol 8819 (Blockeel H, van Leeuwen M, Vinciotti V, editors), pp 37-48. Cham, Switzerland: Springer International Publishing.

Buzsáki G (2004) Large-scale recording of neuronal ensembles. Nat Neurosci 7:446-451. CrossRef Medline

Confais J, Kilavik BE, Ponce-Alvarez A, Riehle A (2012) On the anticipatory pre-cue activity in motor cortex. J Neurosci 32:15359-15368. CrossRef Medline

Denker M, Roux S, Lindén H, Diesmann M, Riehle A, Grün S (2011) The local field potential reflects surplus spike synchrony. Cereb Cortex 21: 2681-2695. CrossRef Medline

Dickey AS, Suminski A, Amit Y, Hatsopoulos NG (2009) Single-unit stability using chronically implanted multielectrode arrays. J Neurophysiol 102:1331-1339. CrossRef Medline

Diesmann M, Gewaltig MO, Aertsen A (1999) Stable propagation of synchronous spiking in cortical neural networks. Nature 402:529-533. CrossRef Medline

Gray CM, König P, Engel AK, Singer W (1989) Oscillatory responses in cat visual cortex exhibit inter-columnar synchronization which reflects global stimulus properties. Nature 338:334-337. CrossRef Medline

Grün S (2009) Data-driven significance estimation of precise spike correlation. J Neurophysiol 101:1126-1140. CrossRef Medline

Han J, Pei J, Yin Y, Mao R (2004) Mining frequent patterns without candidate generation: a frequent-pattern tree approach. Data Mining Knowledge Discovery 8:53-87. CrossRef

Hatsopoulos NG, Ojakangas CL, Paninski L, Donoghue JP (1998) Information about movement direction obtained from synchronous activity of motor cortical neurons. Proc Natl Acad Sci U S A 95:15706-15711. CrossRef Medline

Hatsopoulos N, Joshi J, O’Leary JG (2004) Decoding continuous and discrete motor behaviors using motor and premotor cortical ensembles. J Neurophysiol 92:1165-1174. CrossRef Medline

Ito J, Maldonado P, Singer W, Grün S (2011) Saccade-related modulations of neuronal excitability support synchrony of visually elicited spikes. Cereb Cortex 21:2482-2497. CrossRef Medline

Izhikevich EM (2006) Polychronization: computation with spikes. Neural Comput 18:245-282. CrossRef Medline

Jackson A, Gee VJ, Baker SN, Lemon RN (2003) Synchrony between neurons with similar muscle fields in monkey motor cortex. Neuron 38: 115-125. CrossRef Medline

Kaufman L, Rousseeuw P (1987) Clustering by means of medoids. In: Statistical data analysis based on the L1 norm and related methods (Dodge Y, ed), pp 405-416. New York: Elsevier.

Kilavik BE, Roux S, Ponce-Alvarez A, Confais J, Grün S, Riehle A (2009) Long-term modifications in motor cortical dynamics induced by intensive practice. J Neurosci 29:12653-12663. CrossRef Medline

Kwan HC, Murphy JT, Wong YC (1987) Interaction between neurons in precentral cortical zones controlling different joints. Brain Res 400: 259-269. CrossRef Medline

Louis S, Gerstein GL, Grün S, Diesmann M (2010) Surrogate spike train 
generation through dithering in operational time. Front Comput Neurosci 4:127. CrossRef Medline

Milekovic T, Truccolo W, Grün S, Riehle A, Brochier T (2015) Local field potentials in primate motor cortex encode grasp kinetic parameters. Neuroimage 114:338-355. CrossRef Medline

Murthy VN, Fetz EE (1996) Synchronization of neurons during local field potential oscillations in sensorimotor cortex of awake monkeys. J Neurophysiol 76:3968-3982. Medline

Ochiai A (1957) Zoogeographical studies on the soleoid fishes found in Japan and its neighboring regions. Bull Jpn Soc Sci Fish 22:526-530. CrossRef

Picado-Muiño D, Borgelt C, Berger D, Gerstein G, Grün S (2013) Finding neural assemblies with frequent item set mining. Front Neuroinform 7:9. CrossRef Medline

Pipa G, Riehle A, Grün S (2007) Validation of task-related excess of spike coincidences based on neuroxidence. Neurocomputing 70:2064-2068. CrossRef

Pipa G, Wheeler DW, Singer W, Nikolić D (2008) NeuroXidence: reliable and efficient analysis of an excess or deficiency of joint-spike events. J Comput Neurosci 25:64-88. CrossRef Medline

Riehle A (2005) Preparation for action: one of the key functions of the motor cortex. In: Motor cortex in voluntary movements: a distributed system for distributed functions (Riehle A, Vaadia E, eds), pp 213-240. Boca Raton, FL: CRC.

Riehle A, Requin J (1989) Monkey primary motor and premotor cortex: single cell activity related to prior information about direction and extent of an intended movement. J Neurophysiol 61:534-549. Medline

Riehle A, Grün S, Diesmann M, Aertsen A (1997) Spike synchronization and rate modulation differentially involved in motor cortical function. Science 278:1950-1953. CrossRef Medline

Riehle A, Wirtssohn S, Grün S, Brochier T (2013) Mapping the spatiotemporal structure of motor cortical LFP and spiking activities during reach-to-grasp movements. Front Neural Circuits 7:48. CrossRef Medline

Rubino D, Robbins KA, Hatsopoulos NG (2006) Propagating waves mediate information transfer in the motor cortex. Nat Neurosci 9:1549-1557. CrossRef Medline
Rudolph M, Destexhe A (2003) Tuning neocortical pyramidal neurons between integrators and coincidence detectors. J Comput Neurosci 14: 239-251. CrossRef Medline

Schwarz DA, Lebedev MA, Hanson TL, Dimitrov DF, Lehew G, Meloy J, Rajangam S, Subramanian V, Ifft PJ, Li Z, Ramakrishnan A, Tate A, Zhuang KZ, Nicolelis MA (2014) Chronic, wireless recordings of largescale brain activity in freely moving rhesus monkeys. Nat Methods 11: 670-676. CrossRef Medline

Shimazaki H, Amari S, Brown EN, Grün S (2012) State-space analysis of time-varying higher-order spike correlation for multiple neural spike train data. PLoS Comput Biol 8:e1002385. CrossRef Medline

Smith WS, Fetz EE (2009) Synaptic interactions between forelimb-related motor cortex neurons in behaving primates. J Neurophysiol 102:10261039. CrossRef Medline

Softky WR, Koch C (1993) The highly irregular firing of cortical cells is inconsistent with temporal integration of random EPSPs. J Neurosci 13: 334-350. Medline

Takahashi K, Kim S, Coleman TP, Brown KA, Suminski AJ, Best MD, Hatsopoulos NG (2015) Large-scale spatiotemporal spike patterning consistent with wave propagation in motor cortex. Nat Commun 6:7169. CrossRef Medline

Torre E, Picado-Muiño D, Denker M, Borgelt C, Grün S (2013) Statistical evaluation of synchronous spike patterns extracted by frequent item set mining. Front Comput Neurosci 7:132. CrossRef Medline

Vaadia E, Haalman I, Abeles M, Bergman H, Prut Y, Slovin H, Aertsen A (1995) Dynamics of neuronal interactions in monkey cortex in relation to behavioural events. Nature 373:515-518. CrossRef Medline

Vaidya M, Dickey A, Best M, Coles J, Balasubramanian K, Suminski AJ, Hatsopoulos NG (2014) Ultra-long term stability of single units using chronically implanted multielectrode arrays. Conf Proc IEEE Eng Med Biol Soc 2014:4872-4875. Medline

Wise SP, Weinrich M, Mauritz KH (1983) Motor aspects of cue-related neuronal activity in premotor cortex of the rhesus monkey. Brain Res 260:301-305. CrossRef Medline 